\title{
Privacy Respecting Targeted Advertising for Social Networks
}

\author{
Christian Kahl $^{1}$, Stephen Crane ${ }^{2}$, Markus Tschersich ${ }^{1}$, and Kai Rannenberg ${ }^{1}$ \\ ${ }^{1}$ Goethe University Frankfurt, Chair of Mobile Business \& Multilateral Security, \\ Grüneburgplatz 1, 60629 Frankfurt am Main, Germany \\ ${ }^{2}$ Hewlett-Packard Labs, Long Down Avenue, Stoke Gifford, BRISTOL BS34 8QZ UK \\ \{Christian.Kah1, Markus. Tschersich, \\ Kai.Rannenberg,PICOS\}@m-chair.net, Stephen.Crane@hp.com
}

\begin{abstract}
Online Social Networks form an increasingly important part of people's lives. As mobile technologies improve accessibility, concerns about privacy and trust are more apparent as advertising becomes a critical component of most social network's economic model. In this paper we describe the PICOS project's research into privacy preserving advertising options for social networks. We introduce an architecture that includes new concepts and technologies specifically designed to improve privacy and trust as well as advertising opportunities within social networks.
\end{abstract}

Keywords: Communities, Identity Management, Mobile Advertising, Mobile Marketing, Mobile Social Networks, Privacy, Trust.

\section{Introduction}

Online social networks ${ }^{1}$ such as Facebook, MySpace, and LinkedIn, provide communication services that support the activities of virtual and real world communities (cf. [1], [2], [3]). Nowadays people spend increasing amounts of work and leisure time in using these services for professional and private collaboration and communication purposes. Mobile communication also allows the provision of services that make use of context information (e.g., location, time), thereby enabling a deeper integration of peoples' virtual (mobile) and real world communities (e.g., Loopt, Junaio, match2blue $)^{2}$. Advertising, as a specific marketing activity, is an important way for social network providers to generate revenues, and is hence an integral part of many providers' business models. However, while classical online display advertising is focused on the more general target groups, with advertising activities in social networks often lacking success [2, 22, 23], advertisers are now looking for greater assurance that targeted audiences will be interested in their offerings. Social networks are especially attractive for targeted advertising and viral marketing campaigns [18].

\footnotetext{
${ }^{1}$ Also referred to as "social communities". If not stated otherwise, both terms are used synonymously in this paper.

${ }^{2}$ www.loopt.com, www.junaio.com, www.match2blue.com
} 
However, the inclusion of personal information regarding users in these activities raises questions about user privacy and the use of their personal data. The desire by users for privacy within social networks on the one hand, and the need for advertising in these social networks on the other hand, creates a certain tension between the interests of the involved stakeholders. A balance needs to be achieved between these partially diverging interests of the involved parties, namely users, advertisers and the social network provider [3].

\subsection{Research Question and Approach}

Consequently, a new approach to identity management in social networking services is required in order to meet the stakeholders' different needs. Within the PICOS project $^{3}$, we had the goal to develop such a new approach to identity management that would enhance trust, privacy and identity management aspects of social networking services, while at the same time enabling $3^{\text {rd }}$ party services including marketing and advertising.

PICOS started with a phase of preliminary activities, including the analysis of related contemporary research and an investigation of the context of communities (e.g., legal, technical and economic aspects) (c.f. [9], [10], [3]). Based on requirements of three exemplary mobile communities (anglers, online gamers, taxi drivers), we designed a community platform architecture including concepts to address the gathered requirements [5]. The developed concepts were implemented as prototype community platform and community applications, which we subsequently tested in user trials and evaluated with regard to trust, privacy, usability, ergonomics and legal issues (c.f. [17]).

This paper reports on the PICOS community platform architecture [5], focusing in particular on the targeted advertising component ${ }^{4}$. The following section provides a brief overview of the PICOS architecture and its concepts. Section 3 focuses on the advertising component, as a part of the architecture. Section 4 briefly provides details about the prototypical implementation of the architecture and the advertising component implementation in particular. Section 5 provides an overview of related work and section 6 concludes and indicates aspects for further research.

\section{PICOS Architecture}

The PICOS architecture has been designed to satisfy the needs of several stakeholders, and in so doing minimise the tensions around privacy and trust that would otherwise discourage contributions from any or all parties' involvement in the community. To address the users requirements, the architecture consists of components which provide different functionalities (concepts).

PICOS functionality is delivered as a service. Services could be hosted locally, but in the case of PICOS they are hosted centrally. In this client-server topology, clients (e.g. smart phones) process local services but rely on the social network for shared services and for services that are too demanding (in terms of computing and storage resources) for the client to host.

\footnotetext{
3 The research leading to these results has received funding from the European Community's Seventh Framework Programme (FP7/2007-2011) under grant agreement ${ }^{\circ} 215056$.

${ }^{4}$ For a more general overview of the PICOS architecture, see further [17].
} 


\subsection{Stakeholders}

In situations where personal information is being shared, it is common for the various stakeholders to have different opinions about the use of the data. In social networks these stakeholders are:

- Members/users: The subject of the personal data.

- Community providers: Community service and/or communications provider.

- Community operators: The entity responsible for the operation of the community.

- $\quad 3^{\text {rd }}$ parties: E.g. advertisers, regulators, external service providers.

\subsection{Components and Concepts}

The PICOS architecture comprises a number of new concepts designed to enhance user privacy. The overall intention is to provide users with tools that help them manage their visibility within and outside of the community. The three main categories of concepts are:

Enhanced Identity Management. Based on the concept of mobile identity management [11], the PICOS architecture supports users in managing the disclosure of their current position and mobile identity in communities. E.g. Sub-communities help users in selectively sharing personal information, as they represent a restricted area in which the sharing of content is limited to a sub-group of community members. Partial Identities [12] allow users to create different identities for use in different contexts and purposes. With the help of Partial Identities users are able to have a set of several identities in a single community, and decide for each identity what personal information they want to disclose.

User controlled Information Flows. A balance is needed between revealing (publishing) personal information in order to use functionalities provided by the community, and maintaining a degree of privacy [3]. Location Blurring gives users the enhanced ability to hide their exact position without being completely invisible to others. It foresees the obfuscation of a user's position on a map at various levels. The users can control, who is able to see their exact and their blurred position. Privacy Policies enable users to selectively define policies that control who is allowed to see which kind of personal information.

Privacy Awareness Support. Managing privacy by means of Partial Identities is a complex task. The Privacy Advisor component is designed to provide guidance on privacy related matters that may affect members as they interact with the community. Privacy (and trust) is subjective, and it is often difficult to find a single 'right answer' to questions and concerns about privacy. Hence, the Privacy Advisor is context sensitive and provides hints in specific situations that involve users' personal information (e.g. disclosure of location information, registration and profile management). It warns users when disclosure of information might place their privacy at risk. 


\section{The Advertising Component}

The advertising component enables advertising activities to be carried out with due consideration of context and users' privacy preferences, managed by the aforementioned concepts. The foundation for this component was initially outlined in [24]. Within PICOS we targeted an exemplary application that was extended to include this advertising approach, resulting in a concrete social network service solution that gives users control over the use of their personal data. This unique approach is part of our community platform architecture and within one of our community application prototypes (gaming community prototype).

\subsection{Approach}

Communication can be regarded as one of the main activities conducted in social networks [32]. Hence, in order to include marketing (and in particular advertising) activities into social networks, these activities need to be integrated into the context of the communication processes, in order to be able to receive the attention of the participating users $[24,25]$. Advertising can contribute to the communication in two ways: First, advertisers can provide targeted communication (targeted advertising) to social network users (Business-to-Consumer communication (B2C)). Second, advertisers can support the communication between users (viral marketing) (Consumer-to-Consumer communication (C2C)). While the targeting of advertising activities provides a benefit to the targeted users $[2,33]$, at the same time viral marketing is used in existing social networks ${ }^{5}$ to benefit from the intensive social interactions between users. By supporting both in a novel way, communication between marketers and users is tailored more to an individual user's needs and is consequently more relevant. Further, users are encouraged to communicate with each other about such relevant advertisements $[20,21]$.

\subsection{Component Elements}

To support $\mathrm{B} 2 \mathrm{C}$ and $\mathrm{C} 2 \mathrm{C}$ communication processes, the social network provider acts as an intermediary between advertisers and users. This ensures that personal data of users is neither given to $3^{\text {rd }}$ parties nor that $3^{\text {rd }}$ parties have any direct access to it. Instead, the social network provider (e.g. a game developer in the case of the gaming community example) serves both the advertisers and the consumers, while respecting their specific interests (e.g. privacy of users).

Support of B2C communication. In order to support direct communication between advertiser and user, the social network provider needs to identify the users for which a particular advertisement might be relevant. He thereby conducts a matching operation between the users (consumers) and advertisers (represented by advertisements), based on profile, context and communication information. More targeted advertisements generally receive more attention by users, especially with regard to mobile usage scenarios [27, 36]. On the other hand, users can set their privacy preferences (e.g. using privacy policies and blurring settings) in response to the purpose of targeted

\footnotetext{
${ }^{5}$ See e.g. Facebook Advertising (www.facebook.com/advertising).
} 
advertising. This enables social network provider to respect privacy preferences and satisfy users' needs for relevant advertisements, while at the same time enabling advertisers' to fulfil their interests and reach their target audience.

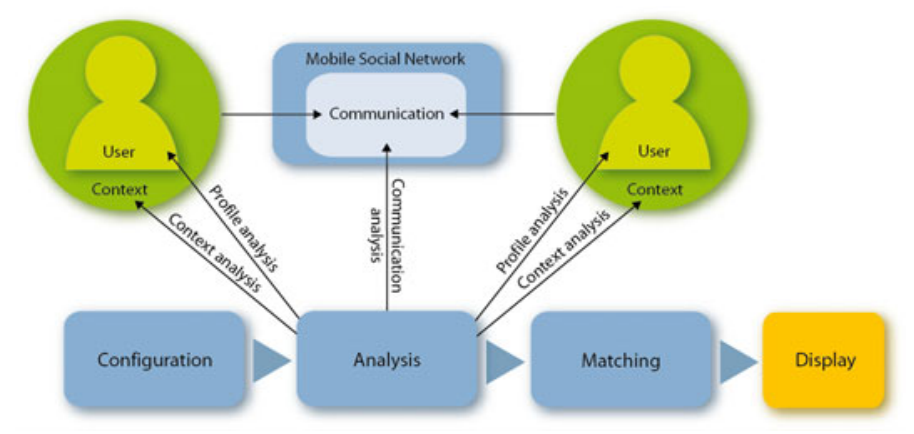

Fig. 1. The process to support targeted advertising (B2C) (Based on [24])

The whole process of supporting B2C communication can be divided into four steps, which are reflected in the design of the PICOS advertising component as follows (Figure 1).

Configuration. The advertising component provides a graphical interface, which allows configuring different advertising activities. As similarly described in e.g. [28, $29,35]$ the advertiser can configure, which message he wants to deliver and whom he wants to target. Hence, the dimensions the advertiser needs to configure are the advertisement itself and the target profile. The form of an advertisement can be a selection of different types, e.g. banners, pop-up, etc. By defining the attributes of the target profile, the advertiser can describe his targeted users. Example: Target Profile: "male, 20-35 years, within $2 \mathrm{~km}$ around my shop, between $12 \mathrm{~h}$ and $18 \mathrm{~h}$, key word in communication: "lunch""

The advertiser can configure how many attributes need to be equal in order to achieve a "matching" of target profile and user profile. For each attribute, the advertiser can also configure if this attribute needs to match in every case. In such a situation, no matching can be achieved if these "necessary" attributes are not fulfilled. E.g., if a user needs to be at least 18 years of age in order to receive an advertisement.

Analysis. In order to determine which advertisement might be relevant for which user, information about the user is needed, which is gathered from the user profile, the context and communications/interactions with other users [24]. The user profile contains attributes such as age, interests and favourite locations. The context is mainly described by the current location of the user (as geo-coordinates) in combination with the time and information which might be derived from the location (e.g. current weather conditions at this location). Communications could be all kinds of interactions in which a user communicates with other users, e.g. directly (mailing or chatting) as well as indirectly (e.g. via contributions in sub-communities). Such additional information, which has not been combined in previous approaches in a similar way, allows a more precise characterization of users and to draw conclusions about what 
users are doing, in addition to who they are. The gathered information leads to a dynamic user profile, which contains the profile, the context and communication information about the user.

Matching. The dynamic user profile characterises the user in his current context. In the "Matching" process the dynamic user profile and the target profiles are compared.

There are different ways to realise such matching in an actual implementation, and which of these ways is chosen might depend on various economic, organisational or technical reasons. In our case a comparison of attributes is conducted. If a pre-defined number of attributes are equal, a match is given. In the approach described by [24], the matching additionally contains a comparison between the communicating users. This is to identify similarities and common interests between them and present matching advertisements not only to one but to both of them.

Display. In the final step of the process, the actual advertisement needs to be shown to the identified matching users. In practice this also includes further considerations regarding the users' device. It might be necessary to adapt the advertisement due to technical specifications or limitations of particular devices and/or operating systems.

Support of C2C communication. The support of C2C communication is based on the principle of viral marketing, that is to initiate a marketing message and let it spread from one user to other users (who distribute it further) like a virus [cf. 18]. The goal here is to establish and support such a viral (marketing) process.

In literature and practice there is a varying understanding about how viral marketing works in detail [26]. In many social networks viral marketing is conducted by introducing a product or brand to the community (e.g. with a related profile or group on Facebook). The difference in our case is that viral marketing is designed to work in a targeted way, in order to address several opinion leaders who further spread the message [16]. This process comprises the following steps:

Configuration. As in $\mathrm{B} 2 \mathrm{C}$ support the advertiser has various options to configure an advertisement and to describe the targeted users, including targeted characteristics (e.g. age, interests). The configuration also includes options regarding the form of the delivery (e.g. pop-up, text message, etc.).

The distinctiveness is that the advertiser defines the characteristics of the "key users", which should be addressed in order to further spread the advertisement. These users are regarded as opinion leaders, which have a stronger influence on their social surrounding $[21,26]$. Depending on the actual advertisement which shall be delivered, there are different definitions of who the "key users" are, e.g. users who are very active with regard to communication, or users who have many relationships to other users (friends) or certain characteristics (e.g. a certain age). The definition of key users might also be a combination of such different characteristics.

Analysis \& Matching. The analysis of user information (profile, context, communication) leads to a dynamic user profile, consisting of profile, context and communication information, as described previously. In the "matching" step, the characteristics of the key users (target profile) are compared to the dynamic profile of a user. The difference 
to the Matching process for targeted advertising is that only a limited number of matching users are addressed, namely the key users. These users are the users which match best with the target profile and fulfil the mentioned characteristics of key users.

Seeding. This phase includes the actual delivery of the advertising message to the identified key users, the so-called "seeding", in order to allow them to pass on the delivered message. Depending on how an advertisement is configured the form of delivery may vary. To support the action of forwarding (spreading) of the delivered message, advertisements need to contain a possibility to immediately and easily share them with other users (e.g. context Link on a specific site, Banner with possibility to forward, etc.).

Triggering. The whole viral marketing process is intended towards the viral distribution of the advertisement. Hence, an important part in this approach is not only to identify adequate users and provide them with the advertising message, as described before, but also to provide or support a motivation to these Users to forward advertisements they receive [30]. One step to support this is already the targeting itself, considering that we aim to provide only highly relevant advertisements to users. Further, an already existing intrinsic motivation of users to forward advertised messages, can be supported by the availability of technical possibilities, which allow and simplify a further recommendation to other users (e.g. "forward" button).

\section{Prototypical Implementation}

The PICOS architecture is a service oriented architecture consisting of 1) access and user management features, and 2) service delivery features. The current $\mathrm{J}_{2} \mathrm{ME}^{6}$ based embodiment consists of a set of hosted web-based services, which implement the described concepts.

The advertising component is implemented based on the example of "commercial points of interest" (CPOI), e.g. cafés, shops, etc. For these CPOI's, a target user profile can be defined for some exemplary attributes (e.g. age). A matching is realised by comparing the attributes between this profile and profiles of users within a certain proximity of the CPOI. Under consideration of users' privacy preferences, advertisements are provided to matching users. The PICOS Privacy policies enable users to select the information which may be used for advertising. A recommendation mechanism further allows supporting user-to-user communication (C2C). Therefore a recommendation button is directly integrated in an advertisement.

\section{Related Work}

The aspect of privacy in online social networks is discussed intensively in the research area (cf. e.g. $[13,14,15])$, but there with focus on online social networks usually not considering the special aspects of mobile social networking services.

${ }^{6}$ Java 2 Platform, Micro Edition (http://www.oracle.com/technetwork/java/javame/overview). 
Other projects, such as PRIME ${ }^{7}$, PrimeLife ${ }^{8}$, PEPERS $^{9}$ and DAIDALOS ${ }^{10}$, have carried out work in this area. However, their work was focused on different aspects, e.g. on privacy and identity management in general (PRIME, PEPERS, DAIDALOS) or privacy in communities but not with regard to a specific application domain (PrimeLife). There are also some concepts in theory and practice which aim to help the user with regard to privacy. For instance, the "Privacy Bird" from AT\&T ${ }^{11}$ advises users on the privacy of website, using a $\mathrm{P}_{3} \mathrm{P}^{12}$ policy matching algorithm. The Trustguide $^{13}$ research confirmed that openness backed up by education, which together provide enhanced understanding and awareness, engenders trust. This is particularly true when applied to situations involving privacy.

Regarding work in relation to the advertising approach, there are a few publications that focus on this aspect with regard to social networks. While some address general aspects, such as business models [4, 25], many focus on the application of viral marketing in the context of communities (e.g. [6, 7, 8, 31]). As mentioned, [24] is concerned with a deeper integration of marketing into the communication processes within social networks, and provides the basis for the advertising approach described in this paper.

\section{Conclusion}

The PICOS Architecture serves as a basis for integrating privacy enhancing concepts and advanced advertising into (mobile) community infrastructures. The architecture enables providers, users and involved 3rd party stakeholders to provide and use privacy enhancing social networking features. The advertising approach adopted by the architecture shows how a deeper integration of advertising is possible in (mobile) social networks and how the tension between diverging interests of the involved stakeholders can be addressed. The prototypical implementation of the PICOS architecture has further shown the feasibility of enclosed concepts such as those focused on in this paper. The currently ongoing analysis and evaluation of the PICOS user trial results will give additional insights on this.

Nevertheless, further research on the integration, usage and benefits of the concepts, and in particular advertising in social networks, remains a challenge for advertisers and social network providers. Much research in this area considers specific aspects of marketing or advertising. However, holistic approaches are needed in order to consider the different stakeholders in social networks and the factors which influence the success of marketing activities. In one of our next steps our research activities will focus on specific application scenarios, considering the diversity of social networks and as well the diversity of products and brands which are subject to marketing.

\footnotetext{
${ }^{7}$ www.prime-project.eu

${ }^{8}$ www.primelife.eu

${ }^{9}$ www.pepers.org

${ }^{10}$ www.ist-daidalos.org

${ }^{11}$ www.privacybird.org/e

${ }^{12}$ www.w3.org/P3P/

${ }^{13} \mathrm{http}$ ://trustguide.org.uk/
} 


\section{References}

1. Nielsen: Critical Mass - Worldwide State of the Mobile Web. Nielsen Mobile (2008)

2. Nielsen: Global Faces and Networked Places - A Nielsen report on Social Networking's New Global Footprint. Nielsen (2009)

3. Liesebach, K., Scherner, T.: D2.4 Requirements. Public Deliverable of EU Project PICOS (2008),

http: / /www.picos-project.eu/Public-Deliverables.29.0.html

4. Hoegg, R., et al.: Overview of business models for Web 2.0 communities. In: Proceedings of Workshop 'Gemeinschaften in Neuen Medien', pp. 33-49. TUD Press, Dresden (2006)

5. Crane, S.: D4.2 Architecture v2. Public Deliverable of EU Project PICOS (2010), http://www.picos-project.eu/Public-Deliverables.29.0.html

6. Leskovec, J., Adamic, L.A., Huberman, B.A.: The Dynamics of Viral Marketing. ACM Trans. Web 1, 1 (2007), Article 5

7. Kempe, D., Kleinberg, J., Tardos, É.: Maximizing the Spread of Influence through a Social Network. In: Proceedings of Ninth ACM SIGKDD International Conference on Knowledge Discovery and Data Mining (SIGKDD 2003), Washington, DC, USA (2003)

8. Hartline, J., Mirrokni, V.S., Sundararajan, M.: Optimal Marketing Strategies over Social Networks. In: Proceedings of the International World Wide Web Conference Committee 2008 (WWW 2008), Beijing, China, April 21-25 (2008)

9. Schrammel, J., Köffel, C., Weiss, S., Kahl, C.: D2.2 Categorisation of Communities. Public Deliverable of EU Project PICOS (2008), http://www.picos-project.eu/Public-Deliverables.29.0.html

10. Kosta, E., Dumortier, J.: D2.3 Contextual Framework. Public Deliverable of EU Project PICOS (2008),

http: / /www.picos-project.eu/Public-Deliverables.29.0.html

11. Müller, G., Wohlgemuth, S.: Study on Mobile Identity Management, Public Deliverable of EU Project FIDIS (2005),

http://www.fidis.net/fileadmin/fidis/deliverables/

fidis-wp3-del3.3.study_on_mobile_identity_management.pdf

12. Hansen, M., Berlich, P., Camenisch, J., Clauß, S., Pfitzmann, A., Waidner, M.: PrivacyEnhancing Identity Management. Information Security Technical Report 9(1), 35-44 (2004)

13. Chew, M., Balfanz, D., Laurie, B.: Undermining Privacy in Social Networks. In: Web 2.0 Security and Privacy (in conj. with IEEE Symposium on Security and Privacy) (2008)

14. Adu-Oppong, F., Gardiner, C.K., Kapadia, A., Tsang, P.P.: Social Circles: Tackling Privacy in Social Networks. In: Proceedings of the 4th Symposium on Usable Privacy and Security (SOUPS 2008), Pittsburgh, Pennsylvania, July 23-25 (2008)

15. Hiltz, S.R., Passerini, K.: Trust and Privacy Concern Within Social Networking Sites: A Comparison of Facebook and MySpace. In: Proceedings of AMCIS 2007 (2007)

16. Dobele, A., Lindgreen, A., Beverland, M., Vanhamme, J., van Wijk, R.: Why pass on viral messages? Because they connect emotionally. Business Horizons 50(4), 291-304 (2007)

17. Kahl, C., Böttcher, K., Tschersich, M., Heim, S., Rannenberg, K.: How to Enhance Privacy and Identity Management for Mobile Communities: Approach and User Driven Concepts of the PICOS Project. In: Rannenberg, K., Varadharajan, V., Weber, C. (eds.) SEC 2010. IFIP Advances in Information and Communication Technology, vol. 330, pp. 277 288. Springer, Heidelberg (2010)

18. Kotler, P., Armstrong, G.: Principles of Marketing, 11th edn. Prentice-Hall, New Jersey (2006) 
19. Kollmann, T.: E-Venture, 1st edn., Gabler, Germany (2006)

20. Schulz, S., Mau, G., Löffler, S.: Virales Marketing im Web 2.0. In: Kilian, T., Hass, B., Walsh, G. (Hg.) Web 2.0 - Neue Perspektiven im E-Business, pp. 249-268. Springer, Heidelberg (2007)

21. Dobele, A., Toleman, D., Beverland, M.: Controlled infection! Spreading the brand message through viral marketing. Business Horizons 48(2), 143-149 (2005)

22. Study on Social Network Adertisements. Linkshare (2009), http: / /www. netimperative.com/news/2009/august/socialnetwork-ads-2018failing-to-engage-users2 019 (retrieved on 2010-06-08)

23. IDC Report. U.S. Consumer Online Attitudes Survey Results, Part III: Social Networking. IDC (2008), http: / / www.idc.com/getdoc.jsp?containerId=214899 (retrieved on 2010-06-08)

24. Kahl, C., Albers, A.: Towards reasonable Revenue Streams through Marketing in Mobile Social Networks. In: Proceedings of the Multikonferenz Wirtschaftsinformatik (MKWI), Göttingen, Germany (2010)

25. Palmer, A., Koenig-Lewis, N.: An experiential, social network-based approach to direct marketing. Direct Marketing: An International Journal 3(3), 162-176 (2009)

26. Phelps, J.E., Lewis, R., Mobilio, L., Perry, D., Raman, N.: Viral Marketing or Electronic Word-of-Mouth Advertising: Examining Consumer Responses and Motivations to Pass Along Email. Journal of Advertising Research 44(4), 333-348 (2004)

27. Ho, S.Y.: Opportunities and challenges of mobile personalization: An exploratory study. In: Newell, S., Whitley, E.A., Pouloudi, N., Wareham, J., Mathiassen, L. (eds.) Proceedings of 17th European Conference on Information Systems (ECIS 2009), Verona, Italy, pp. 1211-1222 (2009)

28. Albers, A., Kahl, C.: Prototypical Implementation of an Intermediary Platform for Context-sensitive Mobile Marketing Applications. In: Proceedings of the 14th Americas Conference on Information Systems (AMCIS), Toronto, Canada (2008)

29. Commercialisation of Context-sensitive Mobile Attention in Mobile Media Markets - Design Recommendations for Mobile Marketing Providers, Schriften zum Mobile Commerce und zum Mobilfunk, Verlag Dr. Kovač, Hamburg, Germany

30. Pousttchi, K., Turowski, K., Wiedemann, D.G.: Mobile Viral Marketing - Ein State of the Art. In: Bauer, H.H., Dirks, T., Bryant, M.D. (Hrsg.) (eds.) Erfolgsfaktoren des Mobile Marketing. Strategien, Konzepte und Instrumente, pp. S.289-S.304. Springer, Berlin (2008)

31. Subramani, M., Rajagopalan, B.: Knowledge Sharing and Influence in Online Social Networks via Viral Marketing. Communications of the ACM 46(12), 300-307 (2003)

32. Carroll, E.: Success Factors of Online Social Networks. The University of North Carolina, Chapel Hill, USA (2007)

33. Ho, S.Y., Kwok, S.H.: The Attraction of Personalized Service for Users in Mobile Commerce: An Empirical Study. ACM SIGecom Exchanges 3(4), 10-18 (2002)

34. Schmidt, A., Beigl, M., Gellersen, H.-W.: There is more to Context than Location. Computers and Graphics 20, S.893-S.901 (1998)

35. Hristova, N., O'Hare, G.M.P.: Ad-me: Wireless Advertising Adapted to the User Location, Device and Emotions. In: Proceedings of 37th Hawaii International Conference on System Sciences, Hawaii, USA (2005)

36. Beales, H.: The Value of Behavioral Targeting (Study), Networked Advertising Initative (2010) 